\title{
Sedimentation of strongly and weakly charged colloidal particles: Prediction of fractional density dependence
}

\author{
Martin Watzlawek* \\ Institut für Theoretische Physik II, Heinrich-Heine Universität \\ Universitätsstr. 1, D-40225 Düsseldorf, Germany \\ and \\ Gerhard Nägele \\ Fakultät für Physik, Universität Konstanz \\ Postfach 5560, D-78434 Konstanz, Germany \\ (Journal of Colloid and Interface Science 214, 170 (1999))
}

Key Words: Sedimentation, Hydrodynamic Interactions, Charge-stabilized Colloids Short running title: Sedimentation of charged particles

*corresponding author; martin@thphy .uni-duesseldorf .de 


\begin{abstract}
We report on calculations of the reduced sedimentation velocity $U / U_{0}$ in homogenous suspensions of strongly and weakly charged colloidal spheres as a function of particle volume fraction $\phi$. For dilute suspensions of strongly charged spheres at low salinity, $U / U_{0}$ is well represented by the parametric form $1-p \phi^{\alpha}$ with a fractional exponent $\alpha=1 / 3$ and a parameter $p \simeq 1.8$, which is essentially independent from the macroion charge $Z$. This non-linear volume fraction dependence can be quantitatively understood in terms of a model of effective hard spheres with $\phi$-dependent diameter. For weakly charged spheres in a deionized solvent, we show that the exponent $\alpha$ can be equal to $1 / 2$, if an expression for $U / U_{0}$ given by Petsev and Denkov [J. Colloid Interface Sci. 149, 329 (1992)] is employed. We further show that the range of validity of this expression is limited to very small values of $\phi$ and $Z$, which are probably not accessible in sedimentation experiments. The presented results might also hold for other systems like spherical proteins or ionic micelles.
\end{abstract}

\title{
1 Introduction
}

The sedimentation velocity $U$ of interacting colloidal particles depends both on the indirect hydrodynamic interactions (HI) mediated by the suspending solvent, and on the microstructure of the suspension. In equilibrium, the latter is determined by direct potential forces arising for example from the steric repulsion between the particles and from the electrostatic repulsion of overlapping double layers. Different pair potentials $u(r)$ lead to rather different microstructures, and this in turn strongly effects the sedimentation. It is well established by theories and experiments that the sedimentation velocity of a dilute suspension of monodisperse hard spheres is given by

$$
\frac{U}{U_{0}}=1-6.55 \phi+\mathcal{O}\left(\phi^{2}\right)
$$

where $\phi$ is the particle volume fraction, and $U_{0}$ is the sedimentation velocity at infinite dilution [1 7]. On the other hand, the long-ranged electrostatic repulsion occurring in suspensions of charged particles can give rise to a reduction in $U$, as compared to a hard sphere dispersion at the same volume fraction [8 11]. This decrease in $U$ is mainly due to the cumulative backflow of displaced fluid, which becomes particularly effective because the probability of two or more charged particles coming close to each other is very small. Conversely, hard spheres are effectively attracted to each other at small interparticle distances $r \lesssim 3 a$, where $a$ is the radius of the spheres. This can be readily seen from the potential of mean force $w(r)=-k_{B} T \ln g(r)$, where $g(r)$ is the radial distribution function of hard spheres, which shows its maximum value at contact distance $r=2 a$ due to excluded volume effects. Closely spaced particles are mutually exposed 
to the downflow of nearby fluid, dragged along with the sedimenting particles. Consequently, the retardation from backflow is reduced, whereas the influence of near-field HI is enhanced for hard sphere suspensions.

While the reduction in $U$ due to long-ranged electrostatic forces is known since many years, it was not realized until recently that, in particular for deionized charge-stabilized suspensions, $U$ can be significantly smaller than $U_{0}$ even for extremely small volume fractions as $\phi \simeq 10^{-4}$. In fact, in the past the effects of HI have been frequently considered to be negligible for dilute suspensions of charged particles [12,13]. However, recent calculations have clearly demonstrated for these systems that HI is of importance at small $\phi$ not only for sedimentation [14 16], but also for short-time [17, 18 and long-time [19 collective diffusion, and for long-time self-diffusion [20]. Moreover, it was shown theoretically for dilute suspensions of charged colloids without added electrolyte that $U$ follows a non-linear $\phi$-dependence of the parametric form [14, 15, 18]

$$
\frac{U}{U_{0}}=1-p \phi^{\frac{1}{3}}
$$

The numerically calculated coefficient $p \simeq 1.8$ was found to be nearly independent of the (effective) particle charge $Z$, provided that $Z$ is kept large enough to completely mask the hard core of the particles. Experimenal results of sedimentation experiments on charged colloids agree favorable with the scaling-prediction of eq. (2) [16, 21]. Similar non-linear volume fraction dependencies are found for the short-time translational and rotational diffusion coefficients of charged particles [22 24].

The same $\phi$-dependence as found for salt-free fluid suspensions of charged colloids is known to be valid for the sedimentation velocity of dilute ordered arrays of fixed spheres 25 28]. For such arrays, the coefficient $p$ is determined analytically as $p=1.76$ for a $s c$ lattice, and as $p=1.79$ for a $f c c$ or a $b c c$ lattice [25,27]. As discussed in detail in Refs. [14, 15, 18], the main peak position $r_{m}$ of the radial distribution function $g(r)$ for highly charged particles in salt-free suspension scales as $r_{m} \propto \phi^{-1 / 3}$ which is also typical of a crystalline solid. This, in fact, turns out to be relevant for eq. (2) to be valid for strongly repelling particles like charged colloids and for ordered arrays of fixed spheres [14, 15, 18, 26].

In the present article, we analyse the sedimentation velocity of charged colloidal dispersions at low salinity both as a function of $\phi$ and of $Z$. Our numerical results for $U / U_{0}$ with system parameters representing monodisperse modified PMMA particles investigated very recently in sedimentation experiments [21], are well described by eq. (2) if the effective macroion charge is chosen such that $Z>100$. We physically explain the scaling relation eq. (2) in terms of an effective hard sphere (EHS) model by using Wertheims analytical expression [29 for the static structure function of hard sphere dispersions obtained in Percus-Yevick approximation. In this specific form of the EHS model, also the numerically determined value $p \simeq 1.8$ is recovered very accurately. We will further demonstrate 
for weakly charged spheres that $U / U_{0}-1$ displays a volume fraction dependence proportional to $\phi^{1 / 2}$, provided the suspension is completely deionized and $\phi$ is chosen small enough that the radial distribution function of the macroions can be approximated by its zero-density limit. This result, however, turns out to be valid only for extremely small values of $\phi$ and $Z$, which we believe are not accessible in sedimentation experiments.

\section{Theory of sedimentation in charged colloids}

We start this section by summarizing the theoretical method used to calculate the reduced sedimentation velocity $U / U_{0}$ of charged colloidal spheres. Our results for $U / U_{0}$ are based on the effective macroion fluid model of charge-stabilized suspensions [15]. In this model, the effective pair potential $u(r)$ between two charged colloidal particles consists of a hard-core part with radius $a$, and of a longer-ranged screened Coulomb potential $u_{e l}(r)$ for $r>2 a$, i.e.

$$
\beta u_{e l}(r)=2 K a \frac{e^{-\kappa(r-2 a)}}{r} .
$$

Here, $K$ is a dimensionless coupling parameter given by

$$
K=\frac{L_{B}}{2 a}\left(\frac{Z}{1+\kappa a}\right)^{2},
$$

where $L_{B}=\beta e^{2} / \epsilon$ is the so-called Bjerrum length, and $\beta=\left(k_{B} T\right)^{-1}$ is the thermal energy. The suspending fluid is treated as a continuum without internal structure, only characterized by its dielectric constant $\epsilon$. Moreover, $Z$ is the effective charge of a colloidal particle in units of the elementary charge $e$. The screening parameter $\kappa$ is given by

$$
\kappa^{2}=4 \pi L_{B}\left[n|Z|+2 n_{s}\right]=\kappa_{c}^{2}+\kappa_{s}^{2},
$$

where $n_{s}$ is the number density of added 1-1-electrolyte, and $n=3 \phi /\left(4 \pi a^{3}\right)$ is the number density of colloidal particles. Notice that $\kappa$ comprises a contribution $\kappa_{c}$ due to counterions, which are assumed to be monovalent, and a second contribution $\kappa_{s}$ arising from added electrolyte. Eq. (3) is a good approximation for $u(r)$ even for strongly charged colloids and for values of $\kappa a$ significantly larger than one, provided the effective charge number $Z$ is regarded as an adjustable parameter [15].

On the time scales probed by dynamic light scattering and by sedimentation experiments, the effect of $\mathrm{HI}$ on the translational motion of the colloidal particles is embodied in the hydrodynamic diffusivity tensors $\boldsymbol{D}_{i j}^{t t}\left(\boldsymbol{R}^{N}\right) ; i, j=1, \ldots, N$ [12, 13, 15, 30, 31]. The short-hand notation $\boldsymbol{R}^{N}=\left(\boldsymbol{R}_{1}, \ldots, \boldsymbol{R}_{N}\right)$ denotes the configuration of the $N$ spherical particles. Without HI, $\boldsymbol{D}_{i j}^{t t}\left(\boldsymbol{R}^{N}\right)=\delta_{i j} D_{0} \mathbf{1}$, 
where $D_{0}=k_{B} T /(6 \pi \eta a)$ is the Stokesian diffusion coefficient of a particle with radius $a$ in a solvent with viscosity $\eta$, and $\mathbf{1}$ denotes the unit tensor.

The reduced short-time sedimentation velocity $U / U_{0}$ of a macroscopically homogeneous suspension of monodisperse colloidal spheres is then given by the zero-wavenumber limit

$$
\frac{U}{U_{0}}=\lim _{q \rightarrow 0} H(q)
$$

of the so-called hydrodynamic function $H(q)$ [13, 15, 17]. This function is defined as

$$
H(q)=\frac{1}{N D_{0}} \sum_{l, j=1}^{N}\left\langle\hat{\boldsymbol{q}} \cdot \boldsymbol{D}_{l j}^{t t}\left(\boldsymbol{R}^{N}\right) \cdot \hat{\boldsymbol{q}} e^{i \boldsymbol{q} \cdot\left(\boldsymbol{R}_{l}-\boldsymbol{R}_{j}\right)}\right\rangle
$$

with wavevector $\boldsymbol{q}$ of magnitude $q$ and corresponding unit vector $\hat{\boldsymbol{q}}=\boldsymbol{q} / q$. The brackets indicate an equilibrium ensemble average. $H(q)$ can be regarded as a generalized (short-time) sedimentation coefficient of particles exposed to spatially periodic external forces aligned with $\hat{\boldsymbol{q}}$, and derived from a weak potential proportional to $\exp [-i \boldsymbol{q} \cdot \boldsymbol{r}]$ [20, 32].

In principle, one needs to distinguish the short-time sedimentation velocity $U$, defined through eq. (6), from the long-time sedimentation velocity, which is determined in conventional sedimentation experiments. In dilute suspensions, however, when the HI are well described as a sum of pairwise additive interactions, both quantities are identical, since then sedimentation does not perturb the equilibrium microstructure [31, 33, 34]. At larger volume fractions, the microstructure becomes distorted from its equilibrium form, since $n$-body HI with $n \geq 3$ becomes important. This distortion leads to an additional change in the sedimentation velocity. Nevertheless, simulations of hard sphere suspensions show that the differences between the short-time and the long-time sedimentation velocities due to memory effects are rather small [35, 36].

The assumption of pairwise additive HI is justified for the important case of dilute (typically $\phi \leq 0.1$ ) charge-stabilized suspensions at sufficiently low ionic strength, as considered here. For such systems, the particles are kept apart from each other due to their strong electrostatic repulsion. Then the $N$-body diffusivity tensors $\boldsymbol{D}_{i j}^{t t}\left(\boldsymbol{R}^{N}\right)$ are well approximated by the two-body tensors

$$
\boldsymbol{D}_{i j}^{t t(2)}\left(\boldsymbol{R}^{N}\right)=\delta_{i j}\left[\mathbf{1}+\sum_{l=1}^{N}{ }^{\prime} \boldsymbol{A}\left(\boldsymbol{R}_{i}-\boldsymbol{R}_{l}\right)\right]+\left(1-\delta_{i j}\right) \boldsymbol{B}\left(\boldsymbol{R}_{i}-\boldsymbol{R}_{j}\right)
$$

where the term $l=i$ is excluded from the sum. This approximation has been verified in the case of translational and rotational self-diffusion by considering also the leading three-body contribution to HI [22,24], and in case of the sedimentation velocity by comparison with a more elaborate method, known as the lowest order form of the $\delta \gamma$-expansion [14, 37, 38]. The two-body translational mobility tensors $\boldsymbol{A}(\boldsymbol{r})$ and $\boldsymbol{B}(\boldsymbol{r})$ are calculated by means of series expansions in powers of $(a / r)$ 
[39,40]. We only quote the leading terms for further reference

$$
\begin{aligned}
& \boldsymbol{A}(\boldsymbol{r})=-\frac{15}{4}\left(\frac{a}{r}\right)^{4} \hat{\boldsymbol{r}} \hat{\boldsymbol{r}}+\mathcal{O}\left(r^{-6}\right) \\
& \boldsymbol{B}(\boldsymbol{r})=\frac{3}{4}\left(\frac{a}{r}\right)[\mathbf{1}+\hat{\boldsymbol{r}} \hat{\boldsymbol{r}}]+\frac{1}{2}\left(\frac{a}{r}\right)^{3}[\mathbf{1}-3 \hat{\boldsymbol{r}} \hat{\boldsymbol{r}}]+\mathcal{O}\left(r^{-7}\right),
\end{aligned}
$$

with $\hat{\boldsymbol{r}}=\boldsymbol{r} / r$. Using eqs. (77,8), $H(q)$ is expressed in terms of an integral

$$
H(q)=1+n \int d \boldsymbol{r} g(r)[\hat{\boldsymbol{q}} \cdot \boldsymbol{A}(\boldsymbol{r}) \cdot \hat{\boldsymbol{q}}+\hat{\boldsymbol{q}} \cdot \boldsymbol{B}(\boldsymbol{r}) \cdot \hat{\boldsymbol{q}} \cos (\boldsymbol{q} \cdot \boldsymbol{r})],
$$

involving these mobility tensors together with the radial distribution function $g(r)$. In this work, we use eqs. (6, 11) together with the series expansions of $\boldsymbol{A}(\boldsymbol{r})$ and $\boldsymbol{B}(\boldsymbol{r})$ for calculating $U / U_{0}$, by including contributions up to order $(a / r)^{20}$.

When terms only up to $\mathcal{O}\left(r^{-4}\right)$ in the series expansions of $\boldsymbol{A}(\boldsymbol{r})$ and $\boldsymbol{B}(\boldsymbol{r})$ are employed, $U / U_{0}$ is given explicitly as

$$
\frac{U}{U_{0}}=1-\phi\left[5+3 \int_{2}^{\infty} d x x(1-g(x))+\frac{15}{4} \int_{2}^{\infty} d x \frac{g(x)}{x^{2}}\right],
$$

with $x=r / a$.

For charge-stabilized suspensions, it is not necessary to account for many terms in the expansion of the two-body mobility tensors, since the integrals in eq. (11) converge rapidly because $g(r)$ is essentially zero at small interparticle distances. In contrast, many terms are needed for hard spheres. For example, using the zero-density form $g_{0}(r)=\Theta(r-2 a)$ for the radial distribution function of hard spheres, we obtain from eq. (12) that $U / U_{0}=1-p \phi$ with $p=6.87$. Here, $\Theta(x)$ is the unit step function. By including terms only up to $\mathcal{O}\left(r^{-3}\right)$, the result is $p=5.0$, as can be seen from eq. (12), when the last term on the right hand side, arising from the term proportional to $(a / r)^{4}$ in eq. (9) is omitted. On the other hand, if terms up to $\mathcal{O}\left(r^{-20}\right)$ are considered, the result for $p$ is improved to $p=6.54$, which is close to the exact value $p=6.55$, first obtained by Batchelor using tabulated numerical results for the near-field HI [1].

To obtain $U / U_{0}$ for dilute charge-stabilized suspensions, we calculate $g(r)$ in the effective macroion fluid model by using, for simplicity, the well-established rescaled mean spherical approximation (RMSA) [15]. On the basis of the pairwise additivity approximation of the HI combined with the $(a / r)$-expansion of the mobility tensors, henceforth referred to as PA-scheme, $U / U_{0}$ can then be calculated as a function of $\phi$.

\section{Fractional density dependence of $U / U_{0}$}




\subsection{Strongly charged particles}

We show in the following that the exponent $1 / 3$ in eq. (2) and the chargeindependence of the parameter $p$, both found from numerical calculations, can be understood quantitatively in terms of a model of effective hard spheres (EHS), which can be treated analytically.

The EHS model accounts for the most important feature of the radial distribution function $g(r)$ of highly charged particles, namely the so-called correlation hole. For an illustration of that, consider fig. 1, which shows a typical $g(r)$ for a salt-free suspension of strongly charged particles at $\phi=0.08$. Due to the strong electrostatic repulsion between the particles, $g(r)$ has a well developed first maximum, and a spherical region with nearly zero probability of finding another particle. This region is referred to as the correlation hole. For small $\phi$, the correlation hole usually extends over several particle diameters [15, 22]. Therefore, we can approximate the actual $g(r)$ of the charge-stabilized system by the radial distribution function of an effective hard sphere (EHS) system with an effective radius $a_{E H S}>a$ and an effective volume fraction $\phi_{E H S}=\phi\left(a_{E H S} / a\right)^{3}$. The EHS-radius $a_{E H S}$ accounts for the electrostatic repulsion between the particles and can be identified as $a_{E H S}=r_{m} / 2$, where $r_{m}$ is the principal peak position of the actual $g(r)$.

Since the extension of the correlation hole is substantially larger than $a$ for small volume fractions and large particle charges, it is then a good approximation to consider only the leading Oseen-term in $\boldsymbol{B}(\boldsymbol{r})$ (cf. eqs. (9, 10,111)) in calculating the sedimentation velocity. $U / U_{0}$ is then well approximated by

$$
\frac{U}{U_{0}}=1+\frac{3 \phi}{a^{2}} \int_{0}^{\infty} d r r h(r)=1+\frac{3 \phi}{a^{2}} \tilde{H}(s=0) .
$$

Here, $h(r)=g(r)-1$ is the total correlation function and

$$
\tilde{H}(s)=\int_{0}^{\infty} d r r h(r) e^{-s r}
$$

denotes the Laplace transform of $r h(r)$. Next, we approximate $h(r)$ by the total correlation function $h_{E H S}\left(r ; \phi_{E H S}\right)$ of the EHS model, evaluated at the effective volume fraction $\phi_{E H S}$. With this approximation for $h(r)$ used in eq. (13), we readily obtain the result $U / U_{0}=1-p \phi^{1 / 3}$ with a fractional exponent $1 / 3$, and the parameter $p$ given by

$$
p=3 \phi_{E H S}^{2 / 3} \tilde{H}_{E H S}(z=0),
$$

where $\tilde{H}_{E H S}(z)$ is the Laplace transform of $y h_{E H S}\left(y ; \phi_{E H S}\right)$ with $y=r / a_{E H S}$ and $z=s a_{E H S}$.

We notice that $\phi_{E H S}$ and hence $p$ are indeed independent of $\phi$ and $Z(\geq 100)$, provided that $a_{E H S}$ is identified with $r_{m} / 2$. This follows from the fact that, for 
deionized suspensions of strongly charged particles where $\kappa_{c} \gg \kappa_{s}$ holds, $r_{m}$ coincides within $3 \%$ with the average geometrical distance $\bar{r}=a(3 \phi /(4 \pi))^{-1 / 3}$ of two particles. For an illustration of this fact consider the inset of fig. 1, which shows RMSA results for $r_{m} / \bar{r}$ as a function of $\phi$. Thus, the scaling relation $r_{m} \approx \bar{r} \propto \phi^{-1 / 3}$ holds and this gives rise to the exponent $1 / 3$ in eq. (2): By identifying $a_{E H S}$ with $\bar{r}$, we obtain an effective volume fraction $\phi_{E H S}=\pi / 6$ independent of $Z$ and $\phi$.

To obtain a numerical value of $p$ according to eq. (15), we take now advantage of an analytic expression for $\tilde{H}_{E H S}(z)$ given in the Percus-Yevick (PY) approximation [29]. By performing the zero- $z$ limit, we obtain in PY approximation after a straightforward calculation the intermediate result

$$
\tilde{H}_{E H S}(z=0)=-\frac{10-2 \phi_{E H S}+\phi_{E H S}^{2}}{5\left(1+2 \phi_{E H S}\right)}
$$

Substitution of eq. (16) into eq. (15) gives

$$
p=\frac{3}{5} \phi_{E H S}^{2 / 3} \frac{10-2 \phi_{E H S}+\phi_{E H S}^{2}}{1+2 \phi_{E H S}} .
$$

Since $\phi_{E H S}=\pi / 6$, we obtain from eq. (17) a value $p=1.76$ remarkably close to $p=1.80$ as determined from the parametric fit of our numerical PA-result for $U / U_{0}$ (cf. following section).

For an illustration of the replacement of the actual $g(r)$ by the EHS- $g(r)$ within the EHS model, see again fig. 1, which shows besides a typical RMSA$g(r)$ of charge-stabilized particles the corresponding EHS- $g(r)$ obtained in PY approximation for $\phi_{E H S}=\pi / 6$.

We finish this section with two remarks. First, we would like to stress again the close connection between the relation eq. (2), found for highly charged colloidal suspensions, and the corresponding result for ordered arrays of fixed spheres. For both systems, the $\phi^{1 / 3}$-scaling behavior of $U / U_{0}$ is caused by a strong structural correlation of the particles, resulting in the scaling relation $r_{m} \propto \phi^{-1 / 3}$ [14, 15, 18]. Although Saffmann already mentioned the possibility for finding a scaling behavior as eq. (2) for highly correlated fluid suspensions [26], the numerical method descibed here provides the first quantitative results on the sedimentation velocity of charged suspensions, leading to the predicted $\phi^{1 / 3}$-scaling in eq. (2) plus the calculation of the prefactor $p$. It is further important to notice that the scaling relation $r_{m} \propto \phi^{-1 / 3}$ is not valid if significantly large amounts of excess electrolyte are added to the suspension, since then the particle diameter becomes another physically relevant length scale besides the mean particle distance $\bar{r}$. The $\phi$-dependence of $U / U_{0}-1$ at small $\phi$ changes then gradually with increasing $n_{s}$ from a $\phi^{1 / 3}$-dependence to the linear $\phi$-dependence of eq. (11) characteristic for hard spheres [16]. 


\subsection{Weakly charged particles}

It is also of interest to consider the opposite limiting case of dilute suspensions of weakly charged spheres in the framework of the effective macroion fluid model. This case was addressed first by Petsev and Denkov [41, 42]. Suppose $Z$ is so small that

$$
\beta u_{e l}(r=2 a)=K \ll 1,
$$

i.e. the electrostatic repulsion can be treated as a small perturbation of the hard-core repulsion between the particles. For very small $\phi, g(r)$ can then be approximated as

$$
g(r) \simeq g_{0}(r)\left[1-\beta u_{e l}(r)\right]
$$

with $g_{0}(r)=\Theta(r-2 a)$. In other words, $g(r)$ is approximated by its zero-density limit $g(r) \simeq \exp (-\beta u(r))=g_{0}(r) \exp \left(-\beta u_{e l}(r)\right)$, linearized with respect to $\beta u_{e l}(r)$. Substitution of eq. (19) in eqs. (6, 11) gives two additive contributions to $U / U_{0}$, the first one arising from the hard-core part $g_{0}(r)$, and the second one from the electrostatic perturbation of $g(r)$ in eq. (19). Explicitly [41, 42]

$$
\frac{U}{U_{0}} \simeq 1-\phi\left[6.55+6 \frac{K}{\kappa a}\right]
$$

where the first term in the bracket is the result of eq. (1), arising from the hardcore part of $g(r)$. As far as the electrostatic part is concerned, it was argued by Petsev and Denkov [41] that the leading Oseen-term in $\boldsymbol{B}(\boldsymbol{r})$ should give the dominant contribution to $U / U_{0}$. Therefore only the Oseen-term is considered here, leading to the second term in the bracket of eq. (20). We will provide a critical discussion of this approximation in the following section.

Next, we further simplify eq. (20) for the case of vanishing excess electrolyte $\left(n_{s}=0\right)$, where $\kappa a=A \phi^{1 / 2}$ with $A=\left(3|Z| L_{B} / a\right)^{1 / 2}$. When $\phi$ and/or $|Z|$ are sufficiently small so that $A \phi^{1 / 2} \ll 1$, eq. (20) is further simplified to

$$
\frac{U}{U_{0}} \simeq 1-\left[6\left(\frac{L_{B}}{2 a}\right)|Z|^{3}\right]^{\frac{1}{2}} \phi^{\frac{1}{2}}+\mathcal{O}(\phi) .
$$

Thus, we expect the reduced sedimentation velocity of dilute deionized suspensions of weakly charged particles to scale like $U / U_{0}=1-p \phi^{1 / 2}$, with a parameter $p$ depending on the macroion charge $Z$ and on the ratio $L_{B} /(2 a)$.

Notice that the hard core contribution $6.55 \phi$ to $U / U_{0}$ in eq. (20) is omitted against the term proportional $\phi^{1 / 2}$ in proceeding from eq. (20) to eq. (21). Therefore, eqs. (20) and (21) do not become equal to each other for $Z \rightarrow 0$. Whereas eq. (20) reduces to the hard sphere result of eq. (11) for $Z=0$, eq. (21) results in $U=U_{0}$ for vanishing particle charge. Obviously, the range of validity of eq. (21) is restricted to small values of $Z$, but still sufficiently large that the second term in eq. (20) plays the dominant role compared to the hard core contribution $6.55 \phi$. We mention already here that due to the strong approximations made in 
deriving eqs. (20,21), the range of validity of these two equations is restricted to extremely small values of $\phi$ and $Z$ (cf. following section).

For eqs. 20,21) to be valid, it is further assumed that the van der Waals attraction among the particles is negligibly small. Experimentally, this might be achieved by coating the particle surface with a thin layer of polymer chains and/or by matching the solvent refractive index to the refractive index of the colloidal particles. If attractions between the particles are present, not only eqs. (20,21) cease to be valid, but also sedimentation velocities larger than for hard spheres may be measured at the same density [5, 66, 16, 43, 44]. This enhanced sedimentation rate is due to the enhanced probability, as compared to hard spheres, of two particles for being close together, thus leading to a reduced retardation from backflow 33.

\section{Numerical results and discussion}

In this section, we present numerical results for the reduced sedimentation velocity $U / U_{0}$ as a function of the volume fraction $\phi$ and of the particle charge number $Z$. Our PA-scheme calculations of $U / U_{0}$ account, if not stated differently, for two-body contributions to the hydrodynamic mobility tensors up to order $(a / r)^{20}$. The system parameters employed in our calculations are $\epsilon=2.183$ (corresponding to cis-decalin as dispersing fluid at temperature $T=293 \mathrm{~K}$ ), and particle radius $a=695 \mathrm{~nm}$, representing modified PMMA particles investigated very recently in sedimentation experiments [21]. We further use $n_{s}=0$, i.e. the ionic strength is essentially determined by the (monovalent) counterions, which counterbalance the charge of the colloidal particles. Obviously, the dielectric constant $\epsilon$ used in the calculations presented here is rather small, leading to strong long-ranged repulsions between the particles even for small surface charges, which, most probably, are present in any polar organic solvent [45]. Furthermore, for these solvents, residual water can cause strong electric charging of the PMMA particles [21]. We therefore present calculations for both weakly and strongly charged particles. Our calculations for strongly charged particles recover the results obtained in Refs. [14, 15, 18] for different system parameters, demonstrating clearly the independence of the scaling relation eq. (2) from specific system parameters, in particular from the solvent dielectric constant $\epsilon$.

\subsection{Strongly charged particles}

In fig. 2, we show the PA-scheme result for $U / U_{0}$, obtained by choosing an effective charge number $Z=150$ large enough that the physical hard-core radius $a$ of the particles constitutes no relevant physical length scale. This numerical result is perfectly fitted by the form $1-p \phi^{\alpha}$, with $p=1.80$ and $\alpha=0.34 \simeq 1 / 3$. As mentioned before, this is in remarkably good quantitative agreement with our EHS 
model result obtained in PY approximation. As discussed in detail in Ref. [16], adding small amounts of excess electrolyte leads to a significant increase in $U$, and the $\phi^{1 / 3}$-scaling behaviour of $U / U_{0}$ does not hold any more. For comparison, fig. 2 includes also the reduced sedimentation velocity of uncharged hard spheres according to eq. (11). Evidently, the sedimentation velocity of charged particles decreases much faster with increasing $\phi$ than one would expect for hard spheres at the same volume fraction.

As shown in fig. 1, the RMSA- $g(r)$ corresponding to the largest volume fraction $\phi=0.08$ considered in fig. 2 has well developed undulations, with the maximum value approximately located at the mean geometrical particle distance $\bar{r}$. We only quote that the corresponding static structure factor $S(q)$ has its principal peak height well below the range of values $2.8-3.1$, where the system starts to freeze according to the empirical Hansen-Verlet rule [46]. A few comments on the quality of the RMSA input for $g(r)$, used in the PA-scheme calculations, is in order here. It is well known [15] that the RMSA underestimates to some extend the oscillations of $g(r)$ and $S(q)$ in case of strongly correlated systems of highly charged particles. In principle, we could use instead of the RMSA an alternative scheme like the Rogers-Young (RY) scheme [47]. The RY sheme is quite accurate within the effective macrofluid model, but has the disadvantage of being numerically far more involved than the RMSA. Fortunately, the RMSA has been found to give nearly identical results as the RY-scheme, provided that a somewhat larger value of $Z$ is used in the RMSA calculations [15]. We have argued before and will show in the following that the parameter $p$ in eq. (22) is nearly independent of $Z$, typically as long as $Z \geq 100$. Consequently, using the RY-scheme instead of the RMSA for the same value of $Z$ should lead to practically identical results for $U / U_{0}$. We have verified this assertion by explicit RY calculations [48, 49].

In fig. [3, we display PA-scheme results for $U / U_{0}$ versus $\phi$ for various values of $Z$. For $Z=0$, we recover the hard sphere result $U / U_{0}=1-p \phi$ with $p=6.54$. An increase in $Z$ leads to a strong reduction in $U$, with a gradual transition from the linear $\phi$-dependence of $U / U_{0}$ towards the non-linear form of eq. (2), with $p \simeq 1.80$. This figure nicely illustrates that $U / U_{0}$ becomes independent of $Z$ for $Z \geq 100$. For these large values of $Z, r_{m}$ stays practically constant when $Z$ is increased with fixed $\phi[48$.

The effect on the PA-results for $U / U_{0}$ caused by truncating the $(a / r)$-expansion of the two-particle mobilities after various terms of increasing order in $(a / r)$, can be assessed from fig. 专. The solid line represents the full PA-scheme result where all two-body contributions up to $\mathcal{O}\left(r^{-20}\right)$ are accounted for. Nearly identical results for $U / U_{0}$ are obtained, even at $\phi=0.08$, when contributions only up to $\mathcal{O}\left(r^{-4}\right)$ are considered. In contrast, the result for $U / U_{0}$ obtained by accounting only for hydrodynamic contributions up to $\mathcal{O}\left(r^{-3}\right)$ shows clear deviations from the full PA-scheme result at volume fractions $\phi \geq 0.01$. As a conclusion, we can state that for dispersions of highly charged particles it is justified to use a 
truncated far-field expansion of the mobility tensors with only the first terms being included, provided a good static input for $g(r)$ is used. The finding that an expansion up to $\mathcal{O}\left(r^{-4}\right)$ leads already to good results indicates further that hydrodynamic $n$-body contributions to $U$ with $n \geq 3$ are indeed negligibly small in the considered $\phi$-range. For practical purposes, it is therefore suitable to use the simple eq. (12) for calculating the reduced sedimentation velocity for highly charged suspensions.

\subsection{Weakly charged particles}

We discuss now the behaviour of $U / U_{0}$ when $Z$ is small. To be specific, consider first the value $Z=5$. For this charge number, $\beta u_{e l}(r)<\left(L_{B} /(2 a)\right) Z^{2} \simeq 0.5$. This implies that the microstructure is affected not only by the electrostatic repulsion but also by the physical hard core of the particles. In fig. 5, we have redrawn from fig. 3 the PA-scheme result for $U / U_{0}$ with $Z=5$ and RMSA-input for $g(r)$. This graph should be compared with the corresponding result for $U / U_{0}$ obtained from the expression given in eq. (20) (henceforth referred to as PD-result). The PD-result for $U / U_{0}$ is completely different from the corresponding PA-scheme result, in particular at larger $\phi$ where the $\mathrm{PD}-U / U_{0}$ even turns negative. The failure of the PD-expression in describing $U / U_{0}$ arises from the fact that the approximations entering in its derivation, i.e. in particular the approximation eq. (19) for $g(r)$, but also the truncation of the electrostatic contribution to $U / U_{0}$ after the leading Oseen-term, are not justified even for a charge number as small as $Z=5$. The consideration of more terms in the hydrodynamic pair mobility contributions up to $\mathcal{O}\left(r^{-20}\right)$, however, leads only to a small increase in $U / U_{0}$, as can be seen from fig. 5, which further includes the PA-scheme result for $U / U_{0}$ with $g(r)$ approximated by the linearized zero-density form of $g(r)$, eq. (19). Another slight improvement in $U / U_{0}$ is achieved when the non-linearized zero-density form $g(r)=\Theta(r-2 a) \exp \left(-\beta u_{e l}(r)\right)$ is used as input in the PAscheme. In fact, the contact value $\beta u_{e l}(2 a)$ is not small enough for $Z=5$ to fully justify a linearization in $\beta u_{e l}(r)$. Therefore, the main reason for the failure of the PD-expression is due to the significant differences of the actual $g(r)$ at $Z=5$ from its zero-density form already for $\phi \simeq 0.01$.

To obtain further insight in the range of validity of the PD-result eq. (20), we display in fig. 6 results for $U / U_{0}$ for an even smaller particle charge $Z=2$ and volume fractions smaller than $\phi=0.01$. As seen from this figure and from fig. 5, the differences between the RMSA-PA-scheme result for $U / U_{0}$ and the PA-scheme result for using $g(r)=\Theta(r-2 a) \exp \left(-\beta u_{e l}(r)\right)$ or $g(r)=\Theta(r-2 a)\left[1-\beta u_{e l}(r)\right]$ as static input become smaller with decreasing $Z$, but are still significant even for $Z=2$ and $\phi \gtrsim 10^{-3}$. For an explanation of this finding, we refer to fig. 7 , where the radial distribution $g(r)$ obtained from the RMSA and from the zero-density expression $g(r)=\Theta(r-2 a) \exp \left(-\beta u_{e l}(r)\right)$ for $Z=2$ and $\phi=0.005$ are shown. Obviously, there are still small differences between the the zero-density expression 
for $g(r)$ and the corresponding RMSA result even for these small values of $Z$ and $\phi$. We show now that these small differences in $g(r)$ cause the large differences in the results for $U / U_{0}$ as illustrated in fig. 6. For this purpose, we plot in the inset of fig. 0 the function $r(1-g(r))$ as obtained from the radial distribution functions shown in the main figure. As easily seen, the two curves for $r(1-g(r))$, corresponding to the two different inputs for $g(r)$, are remarkably different from each other even up to large distances $r$. It is now crucial to notice that according to eq. (12) it is essentially the function $r(1-g(r))$ and not $g(r)$ itself which appears in the integrand when $U / U_{0}$ is calculated including terms up to $\mathcal{O}\left(r^{-1}\right)$ in the series expansions in eqs. (9, 10). Therefore, the small differences in the two $g(r)$ 's considered in fig. 7 give rise to substancial differences in the corresponding sedimentation velocities shown in fig. 6. We can therefore conclude that it is absolutely important to employ an accurate $g(r)$-input for calculating $U / U_{0}$ in the PA-scheme. Due to the use of the (linearized) zero-density approximation of $g(r)$, the expression given by Petsev and Denkov in eq. (20) does not predict the sedimentation velocity of weakly charged particles correctly.

After having explored the high sensibility of $U / U_{0}$ on the form of the $g(r)$ input used in the PA-scheme, it is now apparent that the second approximation made in deriving eq. (20), i.e. the omission of terms in the far-field expansions of the hydrodynamic mobilities of $\mathcal{O}\left(r^{-3}\right)$ in the electrostatic contribution to $U / U_{0}$, is not valid for the small values of $Z$ and $\phi$ used in figs. 6 and 7. This conclusion follows from fig. 6, when the results for $U / U_{0}$ according to eq. (20) and derived from the PA-scheme using eq. (19) as static input are compared. Although the same approximation is employed for $g(r)$, the two results for $U / U_{0}$ do not agree, since the approximations in truncating the series expansions of the hydrodynamic mobility tensors after the terms of $\mathcal{O}\left(r^{-1}\right)$ and $\mathcal{O}\left(r^{-20}\right)$, respectively, are not the same. On the other hand, we have shown in fig. 1t for highly charged particles that the first few terms in the series expansion of eqs. (99.10) are sufficient to calculate $U / U_{0}$. As pointed out before, this is due to the existence of an extended correlation hole in the $g(r)$ of highly charged particles in deionized suspensions (cf. fig. 1). The correlation hole gives rise to a fast concergence of the integrals in eq. (11). However, in case of the weakly charged particles considered here, there is no correlation hole present, as can be seen, e.g., from fig. 7. Therefore, a large number of terms in the series expansions of eqs. (9, 10) are needed for weakly charged particles to calculate $U / U_{0}$. In fact, as discussed before in the case of hard spheres, the terms of $\mathcal{O}\left(r^{-3}\right)$ and $\mathcal{O}\left(r^{-4}\right)$ in the series expansions in eqs. (9.10) contribute in approximately the same weight to $U / U_{0}$, still remarkably large compared the the leading Oseen-term of $\mathcal{O}\left(r^{-1}\right)$, considered only in the electrostatic contribution in the PD-result eq. (20).

To summarize the last presented results, we have shown that the range of validity of eq. (20) is restricted to values of $\phi$ considerably smaller than $10^{-3}$, where the macroion radial distribution function is described very accurately by its linearized zero-density form. Furthermore, for the PD-result eq. (20) to be hold, 
the effective charge $Z$ has to be small enough that the approximation in eq. (19) can be used to calculate $U / U_{0}$, but still not so small that the actual $g(r)$ is too close to its zero-charge limiting form $g_{0}(r)=\Theta(r-2 a)$. Otherwise, the omission of higher order hydrodynamic terms of $\mathcal{O}\left(r^{-3}\right)$ to the electrostatic contribution in eq. (20) ceases to be a good approximation. These severe restrictions limit the range of validity of eq. (20) to values of the system parameters most probably not accessible in sedimentation experiments. Moreover, the relative differences in $U$ and $U_{0}$ become small in the parameter range where eq. (20) should apply.

Let us now turn to some remarks on the $\phi^{1 / 2}$-scaling of $U / U_{0}-1$ proposed in eq. (21). Since eq. (21) is derived from the PD-result eq. (20), the before discussed restricted range of validity of eq. (20) applies also to eq. (21). Furthermore, in eq. (21), the terms linear in $\phi$ are neglected against the term proportional to $\phi^{1 / 2}$. We have shown in fig. 6 that this additional approximation becomes invalid already at small volume fractions, typically $\phi \gtrsim 10^{-3}$. For larger $\phi$, the two results for $U / U_{0}$ in eq. (20) and eq. (21) start to deviate strongly, since the contributions to $U / U_{0}$ linear in $\phi$ (especially the hard core contribution $6.55 \phi$ ) are no longer small compared to the term proportional to $\phi^{1 / 2}$. We therefore also expect the $\phi^{1 / 2}$-scaling of $U / U_{0}-1$ according to eq. (21) not to be measurable in a sedimentation experiment. Nevertheless, we wish to point out that there is a specific range of particle charges $Z$ where $U / U_{0}-1$ indeed scales as $\phi^{1 / 2}$ for a broad range of volume fractions. This range of $Z$-values is determined when the effective particle charge is subsequently lowered from large values $Z \geq 100$, where $U / U_{0}-1$ scales like $\phi^{1 / 3}$, to $Z=0$, where $U / U_{0}-1$ behaves linearly in $\phi$ according to eq. (目). To demonstate the occurance of a $\phi^{1 / 2}$-dependence of $U / U_{0}-1$, we have redrawn in fig. 8 the RMSA-PA result from fig. 5 for $Z=5$ together with the corresponding results for $Z=6$ and $Z=7$. Obviously, all three curves are well described by the form $U / U_{0}=1-p \phi^{\alpha}$ with a parameter $\alpha$ very close to $1 / 2$. Such a $\phi^{1 / 2}$-dependence of $U / U_{0}$ might indeed be measurable in sedimentation experiments on dispersions with suitably chosen particle charges. However, we stress that the occurance of the parameter $\alpha$ close to $1 / 2$ for a broad range of volume fractions as dipicted in fig. 8 is completely different from the prediction eq. (21), which has been shown to be valid only for very small $\phi$.

Finally, we shortly comment on the dependence of our results on the system parameters $T$ and $\epsilon$, which are held fixed in our calculations. Obviously, these two parameters enter into our calculations mainly by determining the Bjerrum length $L_{B}$, which has a rather large value $L_{B}=26.12 \mathrm{~nm}$ due to the small dielectric constant $\epsilon=2.183$ used in this work. As consequence, also the ratio $L_{B} /(2 a)$ is comparatively large. This ratio determines together with $Z$ the strength of the electrostatic repulsion between the particles. For that reason, one might argue that our dicussion concerning the use of eq. (19) for $g(r)$ in calculating $U / U_{0}$ is not longer valid for smaller values of $L_{B}$. However, explicit PA-scheme calculations for system parameters as used in Ref. [16] and/or a simple estimate show that our conclusions derived above remain valid even for considerably smaller 
values of $L_{B}$ : Consider a value of $L_{B}$ ten times smaller than the one used in the above presented calculations, e.g. a value like in sedimentation experiments on charged silica particles in ethanol [16]. Then, according to eqs. (3, (4), one might choose a particle charge approximately $\sqrt{10} \simeq 3.16$ times larger than in our calculations to achieve the same strength of repulsion between the particles. As shown above for our choice of system parameters, the use of eq. (19) for $g(r)$ is a poor approximation even for low volume fractions $\phi \leq 0.01$ and a particle charge $Z=2$. Therefore, even for a system with $L_{B}$ ten times larger, eqs. (20,21) are applicable only for particle charges considerably smaller than $Z=6$. This slightly extended parameter range for eqs. (20,21) to be valid is most probably still too restricted to be experimentally accessible.

\section{Concluding remarks}

We have presented theoretical results for the reduced sedimentation velocity $U / U_{0}$ of monodisperse charged suspensions in dependence of the volume fraction $\phi$ and of the particle charge number $Z$. Our theoretical model for $U / U_{0}$ is based on the effective macroion fluid model and on the assumption that pairwise additive $\mathrm{HI}$ prevails at sufficiently small $\phi$. The numerical results for $U / U_{0}$ in case of strongly charged particles at low salinity are well parametrized by the form $1-p \phi^{1 / 3}$.

We have shown that the exponent $1 / 3$ and the value of the charge-independent parameter $p=1.8$ can be quantitatively understood in terms of a model of effective hard spheres of radius $a_{E H S}$ which depends on the volume fraction. Using Percus-Yevick input for the static pair correlation function of hard spheres, the EHS model can be treated analytically and leads to a value of $p$ very close to 1.8 .

It was further demonstrated that $U / U_{0}-1$ can scale like $\phi^{1 / 2}$ in case of dilute suspensions of very weakly charged particles. This peculiar volume fraction dependence of $U / U_{0}$ derives from an expression given by Petsev and Denkov [41,42], when it is further assumed that the ionic strength in the system is mainly due to counterions. Our numerical calculations reveal that both the original expression for $U / U_{0}$ given by Petsev and Denkov, and the derived expression showing the $\phi^{1 / 2}$-scaling of $U / U_{0}-1$, are only valid for very small particle volume fractions, which we expect not to be accessible in conventional sedimentation experiments.

\section{Acknowledgements}

We are grateful to Barbara Löhle (University of Konstanz) for providing RogersYoung calculations of various static distribution functions, and to Bruce Ackerson (Oklahoma State University) and Barbara Mandl (formerly University of Konstanz) for helpful discussions. We further thank on of the referees for calling our 
attention to Ref. [26]. M.W. thanks the Deutsche Forschungsgemeinschaft for financial support within SFB 513 and SFB 237. 


\section{References}

[1] G. K. Batchelor, J. Fluid Mech. 52, 245 (1972).

[2] B. Cichocki and B. U. Felderhof, Physica A 154, 213 (1989).

[3] H. J. H. Clerx and P. P. J. M. Schram, J. Chem. Phys. 96, 3137 (1992).

[4] M. M. Kops-Werkhoven and H. M. Fijnaut, J. Chem. Phys. 74, 1618 (1981).

[5] R. Buscall, J. W. Goodwin, R. H. Ottewill, and T. F. Tadros, J. Coll. Int. Sci. 85, 78 (1982).

[6] D. M. E. Thies-Weesie, A. P. Philipse, and H. N. W. Lekkerkerker, J. Coll. Int. Sci. 177, 427 (1996).

[7] W. Xu, A. Nikolov, and D. T. Wasan, J. Coll. Int. Sci. 197, 160 (1998).

[8] C. C. Reed and J. L. Anderson, in Hydrosols and Rheology, edited by M. Kerker, volume 4 of Colloid and Interface Science, Academic Press, New York, 1976.

[9] C. C. Reed and J. L. Anderson, AIChE J. 26, 816 (1980).

[10] I. Snook and W. van Megen, J. Coll. Int. Sci. 100, 194 (1984).

[11] E. Dickinson, J. Coll. Int. Sci. 73, 578 (1980).

[12] P. N. Pusey and R. J. A. Tough, in Dynamic light scattering, edited by R. Pecora, Plenum, New York, 1985.

[13] P. N. Pusey, in Liquids, Freezing and Glass Transition: II, edited by J.-P. Hansen, D. Levesque, and J. Zinn-Justin, North Holland, Amsterdam, 1991.

[14] G. Nägele, B. Steininger, U. Genz, and R. Klein, Physica Scripta T 55, 119 (1994).

[15] G. Nägele, Phys. Rep. 272, 215 (1996).

[16] D. M. E. Thies-Weesie, A. P. Philipse, G. Nägele, B. Mandl, and R. Klein, J. Coll. Int. Sci. 176, 43 (1995).

[17] G. Nägele, O. Kellerbauer, R. Krause, and R. Klein, Phys. Rev. E 47, 2562 (1993).

[18] G. Nägele, B. Mandl, and R. Klein, Progr. Colloid Polym. Sci. 98, 117 (1995).

[19] P. Baur, G. Nägele, and R. Klein, Phys. Rev. E 53, 6224 (1996). 
[20] G. Nägele and P. Baur, Physica A 245, 297 (1997).

[21] B. J. Ackerson, Oklahoma State University, private communication.

[22] M. Watzlawek and G. Nägele, Physica A 235, 56 (1997).

[23] M. Watzlawek and G. Nägele, Prog. Coll. Polym. Sci. 104, 168 (1997).

[24] M. Watzlawek and G. Nägele, Phys. Rev. E 56, 1258 (1997).

[25] H. Hasimoto, J. Fluid Mech. 5, 317 (1959).

[26] P. G. Saffman, Stud. Appl. Math. 52, 115 (1973).

[27] A. A. Zick and G. M. Homsy, J. Fluid Mech. 115, 13 (1982).

[28] J. F. Brady, R. J. Phillips, J. C. Lester, and G. Bossis, J. Fluid Mech. 195, 257 (1988).

[29] M. S. Wertheim, Phys. Rev. Lett. 10, 321 (1963).

[30] R. B. Jones and P. N. Pusey, Annu. Rev. Phys. Chem. 42, 137 (1991).

[31] J. Dhont, An Introduction to Dynamics of Colloids, volume 2 of Studies in interface science (eds. D. Möbius and R. Miller), Elsevier, Amsterdam, 1996.

[32] W. B. Russel and A. B. Glendinning, J. Chem. Phys. 74, 948 (1981).

[33] W. B. Russel, D. A. Saville, and W. R. Schowalter, Colloidal Dispersions, Cambridge University Press, Cambridge, 1989.

[34] A. B. Glendinning and W. B. Russel, J. Coll. Int. Sci. 89, 124 (1982).

[35] A. J. C. Ladd, J. Chem. Phys. 93, 3484 (1990).

[36] A. J. C. Ladd, Phys. Fluids A 5, 299 (1993).

[37] C. W. J. Beenakker and P. Mazur, Physica A 126, 349 (1984).

[38] U. Genz and R. Klein, Physica 171, 26 (1991).

[39] R. B. Jones and R. Schmitz, Physica A 149, 373 (1988).

[40] B. Cichocki, B. U. Felderhof, and R. Schmitz, PhysicoChem. Hydr. 10, 383 (1988).

[41] D. N. Petsev and N. D. Denkov, J. Coll. Int. Sci. 149, 329 (1992).

[42] N. D. Denkov and D. N. Petsev, Physica A 183, 462 (1992). 
[43] P. Y. Cheng and H. K. Schachman, J. Polym. Sci. 16, 19 (1955).

[44] J. W. Jansen, C. G. De Kruif, and A. Vrij, J. Coll. Int. Sci 114, 501 (1986).

[45] A. P. Philipse, Curr. Opinion Coll. Int. Sci. 2, 200 (1997).

[46] J.-P. Hansen and L. Verlet, Phys. Rev. 184, 150 (1969).

[47] F. J. Rogers and D. A. Young, Phys. Rev. A 30, 999 (1984).

[48] M. Watzlawek, Diplom-thesis, University of Konstanz, 1996.

[49] M. Watzlawek, B. Löhle, and G. Nägele, unpublished results. 


\section{Figure captions}

Figure 1: RMSA radial distribution function $g(r)$ (dashed line) for a suspension of charged particles with system parameters $a=695 \mathrm{~nm}, \epsilon=2.183, T=293$ $\mathrm{K}, n_{s}=0$ (i.e. no added salt), $Z=150$, and $\phi=0.08$. Also shown is the radial distribution function of the EHS model in PY approximation (solid line). The effective volume fraction is $\phi_{E H S}=\pi / 6$, corresponding to the identification $2 a_{E H S}=r_{m}=\bar{r}$ (see text). The inset shows the peak position $r_{m}$ of the RMSA$g(r)$ for the same system paramters, normalized by the mean particle distance $\bar{r}$, as function of $\phi$.

Figure 2: PA-scheme result for the reduced sedimentation velocity $U / U_{0}$ of strongly charged particles in a deionized solvent (solid line). The result is well parametrized by the form $1-1.80 \phi^{0.34}$. System parameters as in fig. 11. For

comparison, the dashed-dotted line represents the lowest-order density result for hard spheres according to eq. (11).

Figure 3: PA-scheme results for $U / U_{0}$ for various values of the effective charge number $Z$ as indicated in the figure. All other system parameters as in fig. 11. Notice that $U / U_{0}$ is nearly independent of $Z$ when $Z \geq 100$.

Figure 4: Dependence of $U / U_{0}$ on various two-body contributions to the hydrodynamic mobilities. Included terms of the series expansions in eqs. (9, 10) and used in the PA-scheme as indicated in the figure. System parameters as in fig. 2 . 
Figure 5: $U / U_{0}$ versus $\phi$ for particle charge $Z=5$. Other parameters chosen as in fig. 1. Shown are the PA-scheme results obtained using as static input the RMSA$g(r)$ (solid line), the zero-density form $g(r)=\Theta(r-2 a) \exp \left(-\beta u_{e l}(r)\right)$ (dashed line), and the linearized zero-density form of $g(r)$ according to (19) (dasheddotted line). Further displayed is $U / U_{0}$ corresponding to eq. (20) (dotted line).

Figure 6: $U / U_{0}$ versus $\phi$ for particle charge $Z=2$. All other parameters as in fig. 1. Shown are PA-scheme results obtained using as input the RMSA- $g(r)$ (solid line), and the zero-density form $g(r)=\Theta(r-2 a) \exp \left(-\beta u_{e l}(r)\right)$ (dashed line). The latter is practically identical with the $\mathrm{PA}-U / U_{0}$ calculated with the linearized $g(r)$ of eq. (19). Further displayed are results for $U / U_{0}$ according to eq. (20) (dashed-dotted line) and eq. (21) (dotted line). The thin solid line represents the hard sphere result of eq. (11).

Figure 7: Radial distribution functions $g(r)$ for $Z=2$ and $\phi=0.005$, obtained in the RMSA (solid line) and given by $g(r)=\exp (-\beta u(r))$ (dashed line). Parameters aside from $Z$ and $\phi$ as in fig. 11. The inset shows the corresponding functions $r(1-g(r))$.

Figure 8: RMSA-PA-scheme results for $U / U_{0}$ at particle charges $Z=5, Z=6$, and $Z=7$. All other parameters as in fig. 11. The result for $Z=5$ (solid line) is well parametrized by the form $U / U_{0}=1-2.18 \phi^{0.56}$, whereas the results for $Z=6$ (dashed line) and $Z=7$ (dashed-dotted line) are very well fitted within the shown volume fraction range by $U / U_{0}=1-2.06 \phi^{0.53}$ and $U / U_{0}=1-1.97 \phi^{0.50}$, respectively. 


\section{Watzlawek and Nägele, Fig. 1}

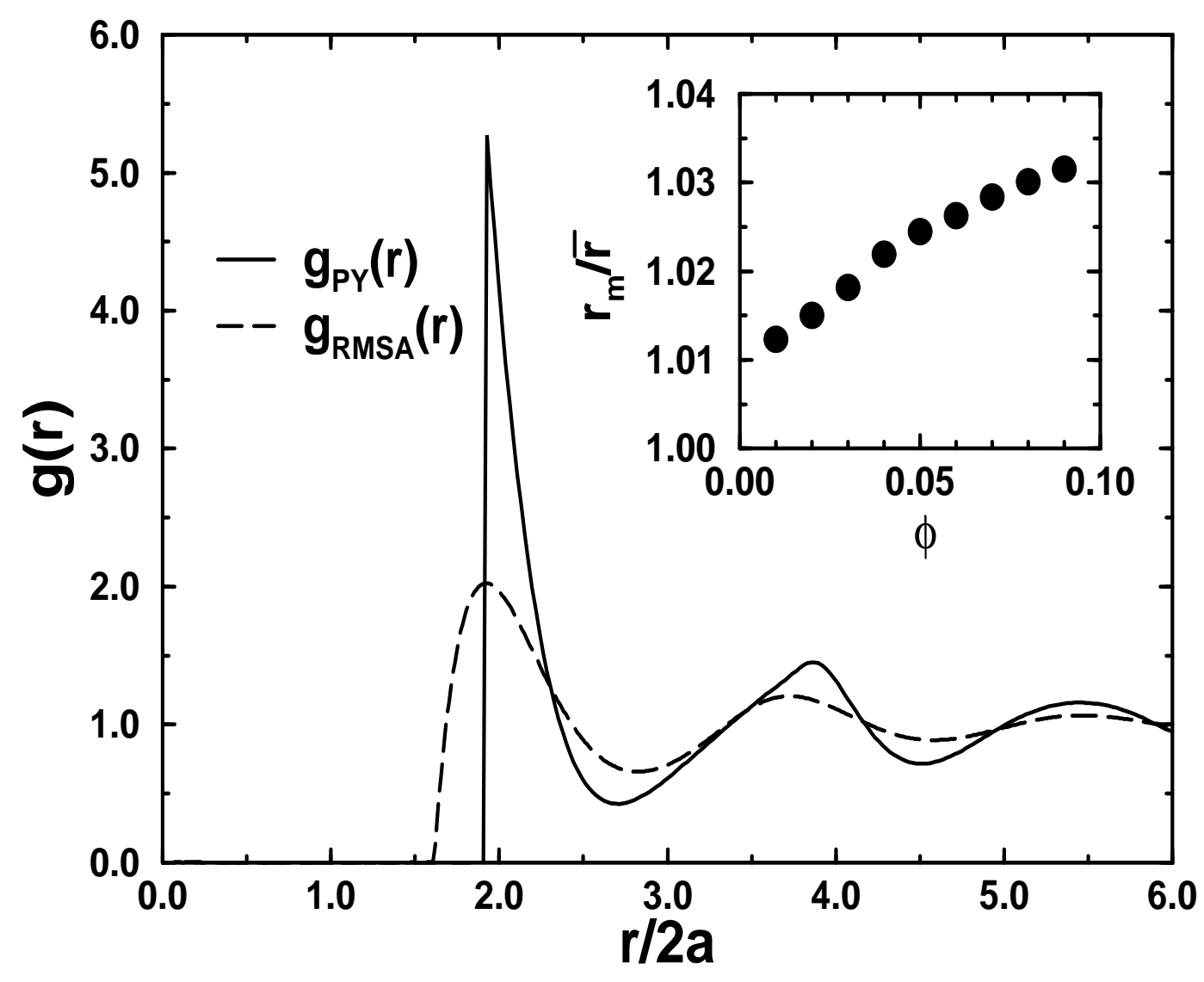


Watzlawek and Nägele, Fig. 2

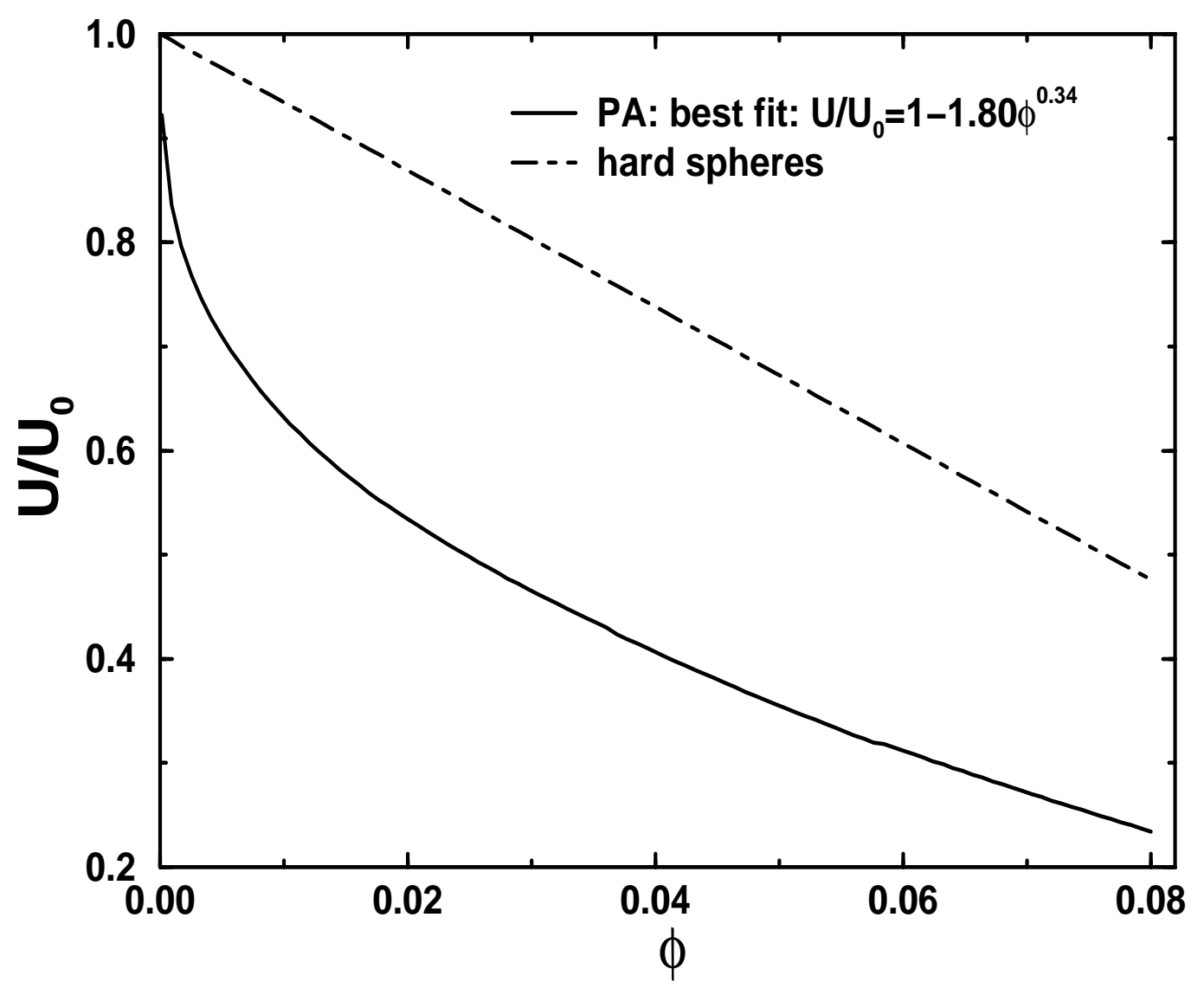




\section{Watzlawek and Nägele, Fig. 3}

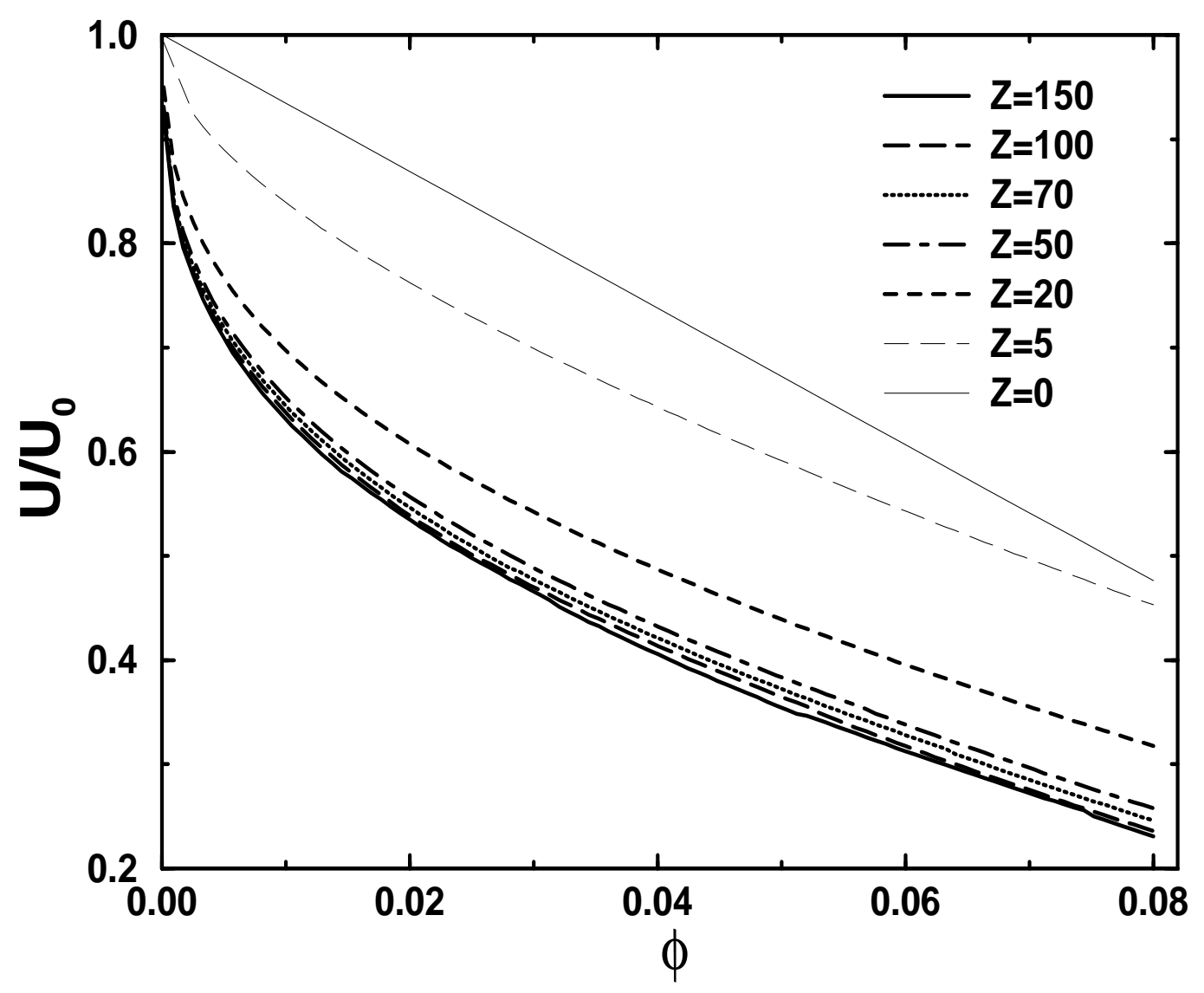


Watzlawek and Nägele, Fig. 4

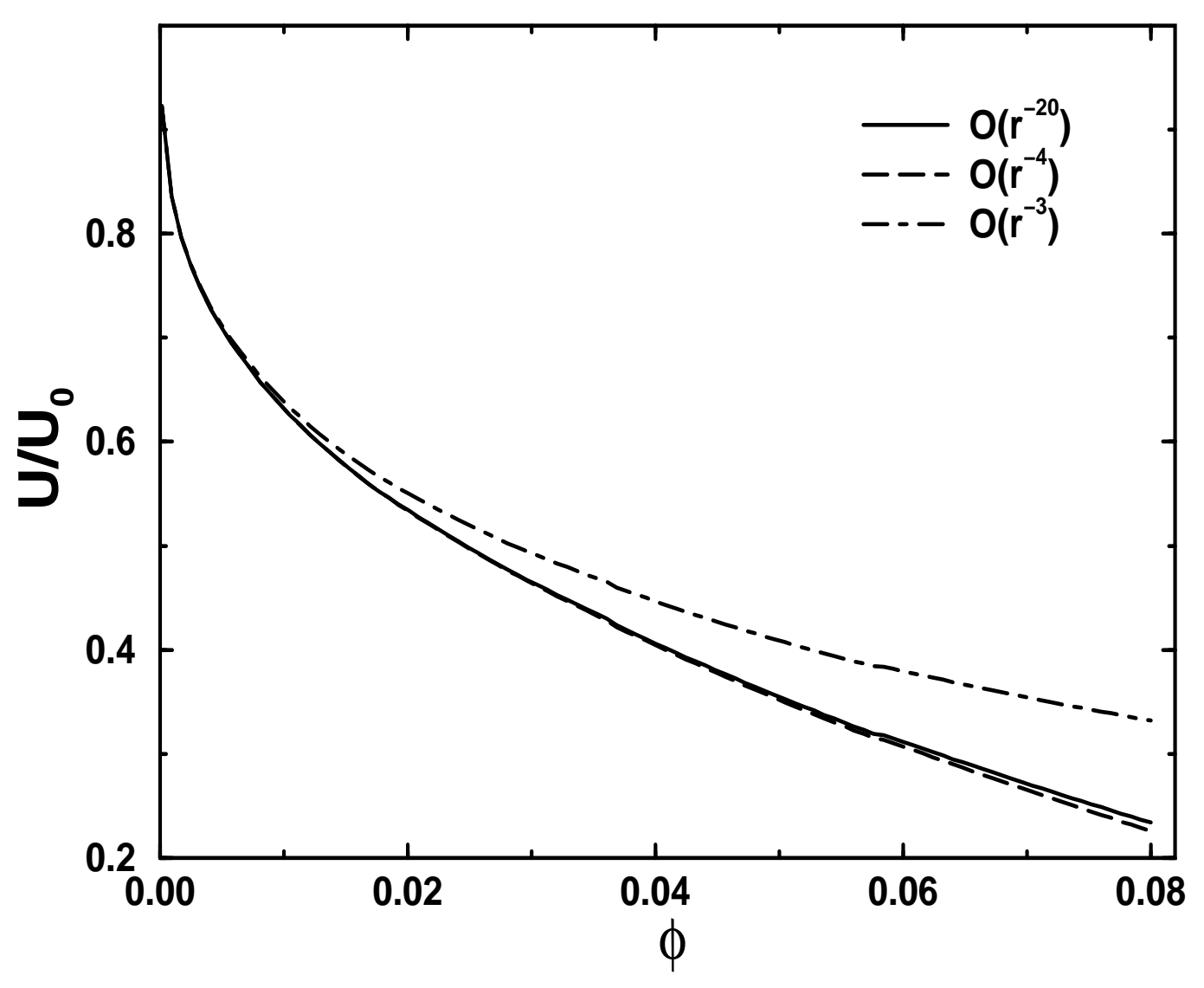


Watzlawek and Nägele, Fig. 5

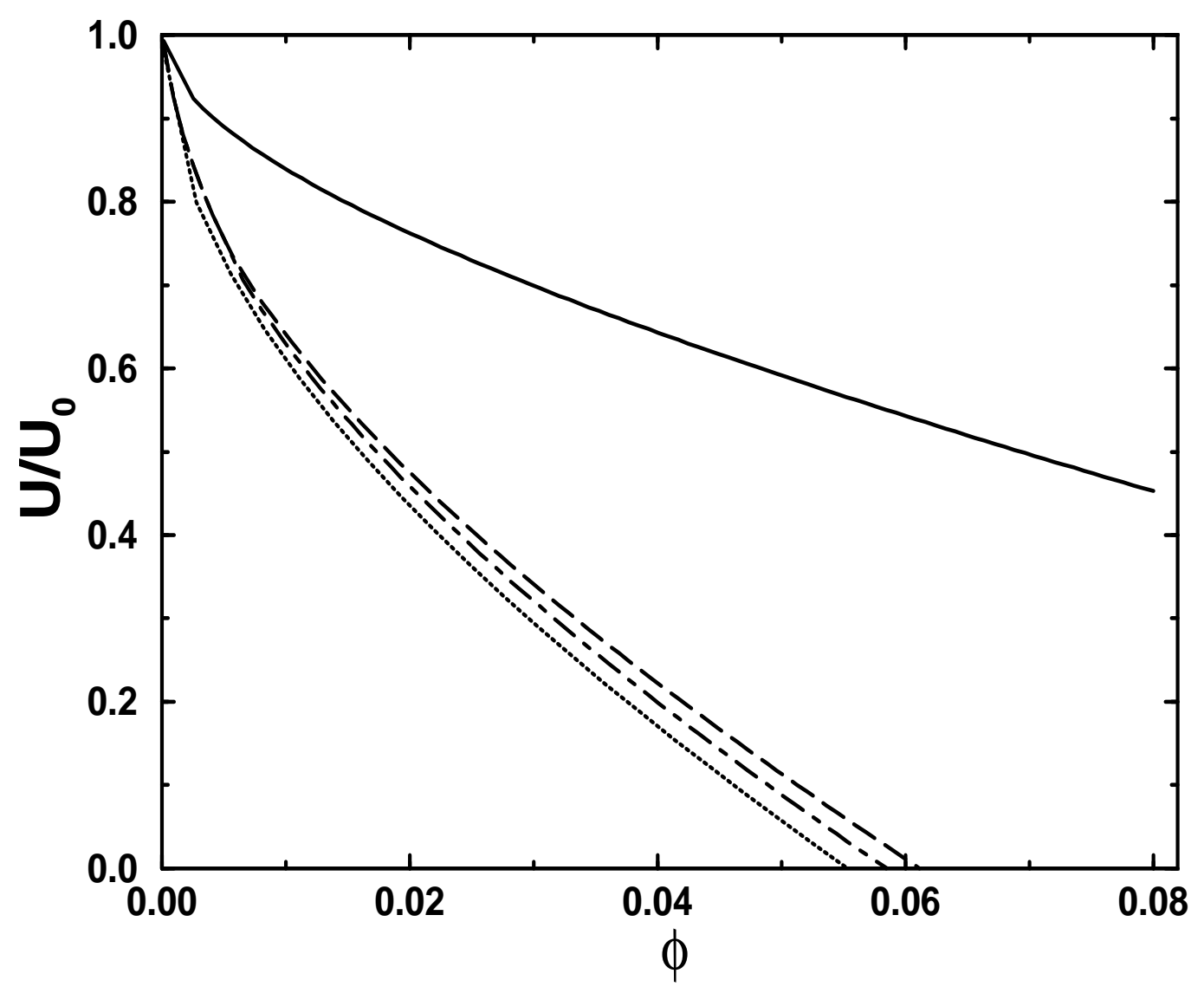




\section{Watzlawek and Nägele, Fig. 6}

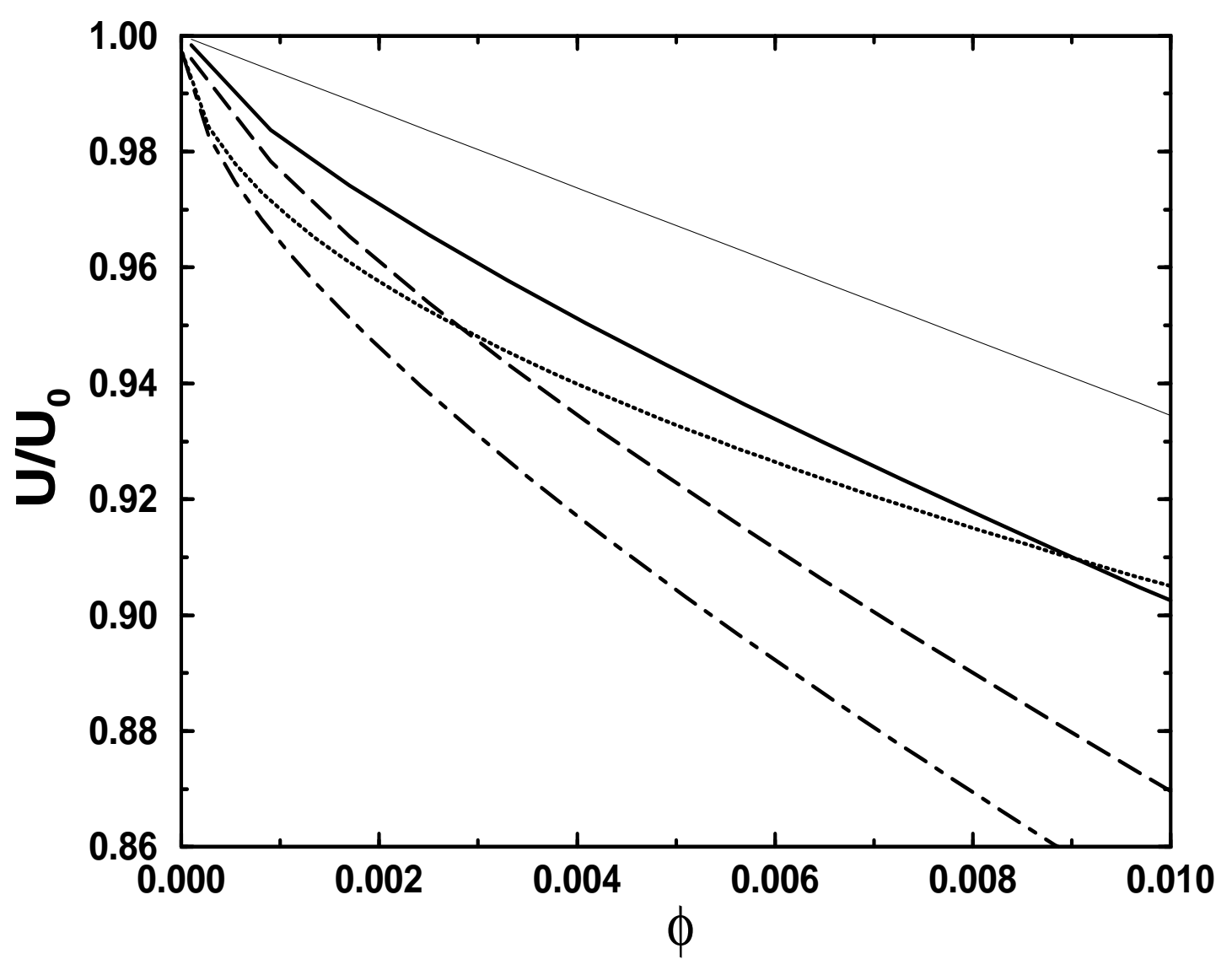


Watzlawek and Nägele, Fig. 7

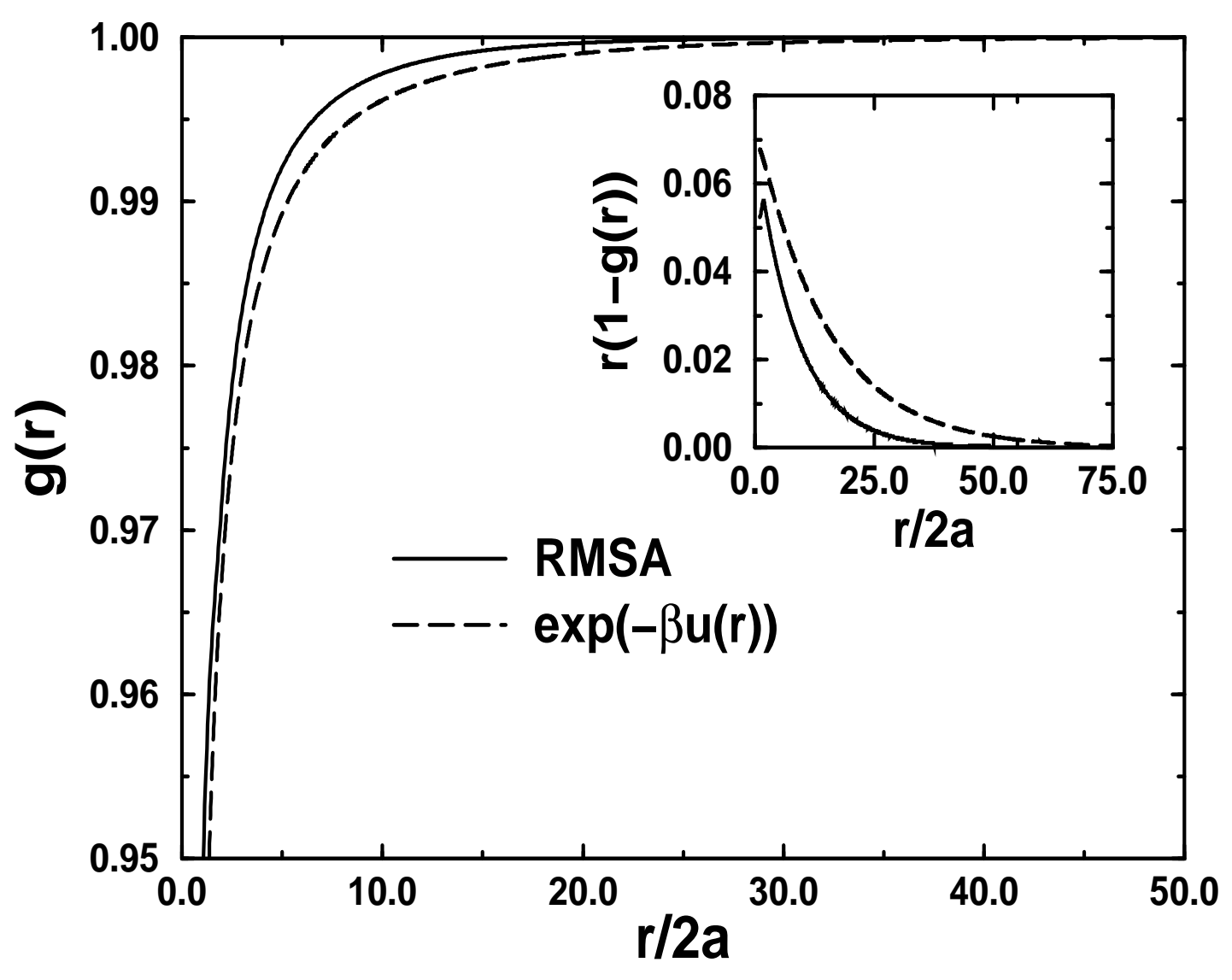


Watzlawek and Nägele, Fig. 8

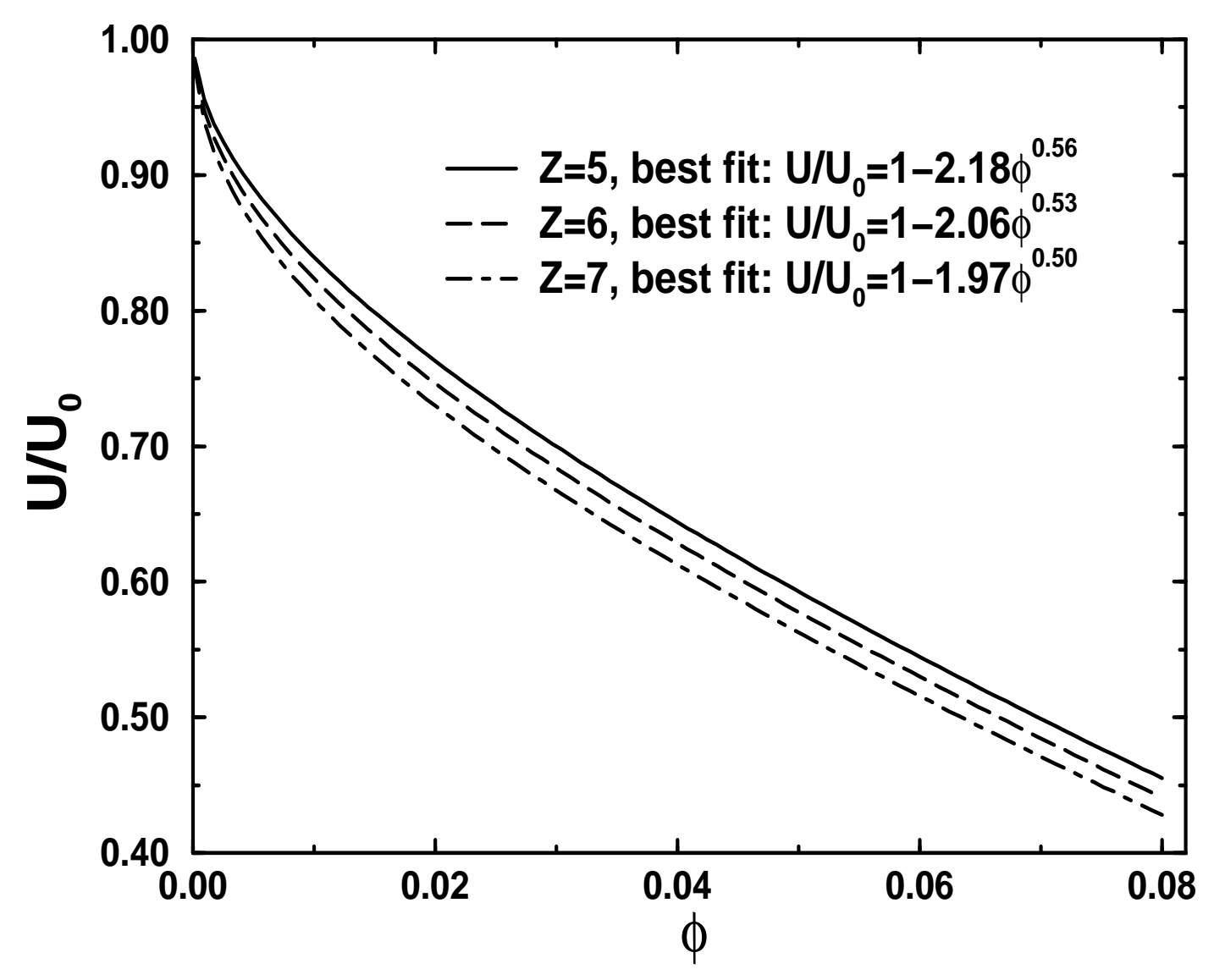

